\title{
2021 Emerging Investigator Issue of Science China Chemistry
}

\author{
Li-Jun $\operatorname{Wan}^{1,2^{*}}$ \\ ${ }^{1}$ Institute of Chemistry, Chinese Academy of Sciences, Beijing 100190, China; \\ ${ }^{2}$ University of Chinese Academy of Sciences, Beijing 100049, China
}

Received October 19, 2021; accepted October 19, 2021; published online October 20, 2021

Citation: Wan LJ. 2021 Emerging Investigator Issue of Science China Chemistry. Sci China Chem, 2021, 64: 1811-1816, https://doi.org/10.1007/s11426-021$1138-9$

In 2020, Sci. China Chem. (SCC) published an "Emerging Investigator Issue," featuring the works conducted by young scientists at an early stage of their independent research careers. The research finding in this issue has showcased the excitement and potential of a new-generation of scientists in pushing forward the development of chemistry and related fields, and has been well-received for its academic value and novel perspective.

On the basis of the success of 2020, SCC has now edited the 2021 Emerging Investigator Issue. The present issue is composed of 21 Reviews and Articles. All 21 young contributors are recommended by SCC Editorial Board Committee and the National Natural Science Foundation Committee. The works covered in this special issue include the research fields of organic synthesis, precise cellular analysis, cell imaging, nanomedicine, electrocatalysis, 2D materials, aggregation-induced emission materials, metalorganic frameworks, aluminum-ion batteries, organic solar cells, as well as perovskites.

Listed below are the profiles for each invited author. We wish all young scientists continued success in their future careers, and we are grateful for their outstanding contributions to SCC.

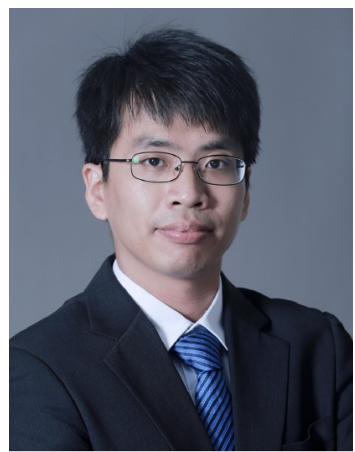

Dr. Chaohua Cui is currently a professor at Soochow University. He received his B.S. degree from Beijing Institute of Technology in 2009, M.S. degree from Beijing University of Chemical Technology (co-supervised by Prof. Yongfang Li at Institute of Chemistry, Chinese Academy of Sciences) in 2011, and his Ph.D. degree in 2014 from Hong Kong Baptist University under the supervision of Prof. Wai-Yeung Wong. He serves as an Associate Editor for Roy. Soc. Open Sci. and a Young Advisory Board Member for InfoMat. He was selected as "Emerging Investigator" by J. Mater. Chem. A (2020), Chem. Commun. (2021), and Sci. China Chem. (2021), respectively. His research focuses on organic photovoltaic materials and devices.

*Corresponding author (email: wanlijun@iccas.ac.cn) 


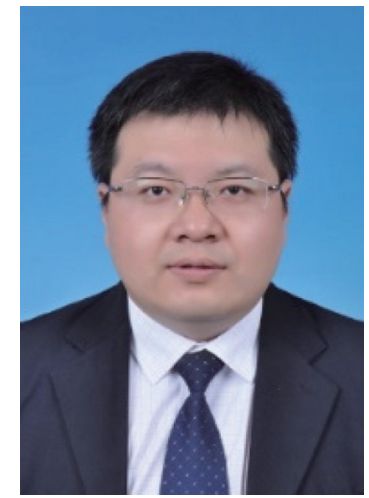

Dr. Zhiqian Guo is a professor at East China University of Science \& Technology (ECUST). He received his Ph.D. degree from ECUST in 2010 under the supervision of Professor Weihong Zhu and then worked with Professor Juyoung Yoon at Ewha Womans University, Korea on organic chemistry from 2011 to 2012. He was a recipient of the National Natural Science Foundation of China (NSFC) for Excellent Young Grants (2016), and Cheung Kong Young Scholar (2017). His current research focuses on functional chromophores, including fluorescent sensors, drug delivery system and molecular logic devices for biomedical application.

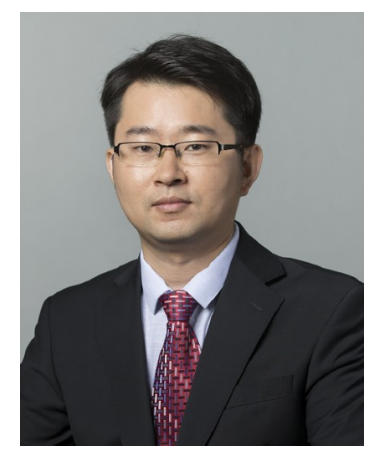

Dr. Guodong Li received his B.S. degree from University of Jinan in 2004, M.S. degree from Ocean University of China in 2007, and Ph.D. degree from Beijing University of Chemical Technology in 2011, respectively. Then, he joined the National Center for Nanoscience and Technology. He was a visiting scholar in Stanford University from 2016 to 2017. His research focuses on design and fabrication of inorganic nanocomposites with well-defined structures as well as their applications in catalysis and energy.

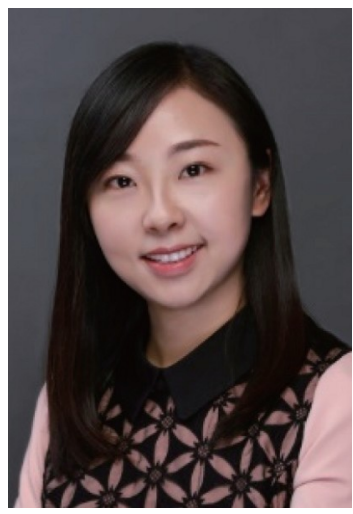

Dr. Xiaoding Lou received her Ph.D. degree in organic chemistry from Wuhan University in 2012 under the supervision of Prof. Zhen Li. Then, she worked as a research associate in Prof. Ben Zhong Tang's group at the Hong Kong University of Science and Technology. In 2013, she joined Huazhong University of Science and Technology as an assistant professor. In 2016, she conducted her work in the group of Prof. Alan J. Heeger at University of California, Santa Barbara as a visiting scholar. Since 2017, she has been a professor of analytical chemistry at China University of Geosciences (Wuhan). Her current research focuses on the design of peptide- or DNA-modified probes and their applications in precise cellular analysis.

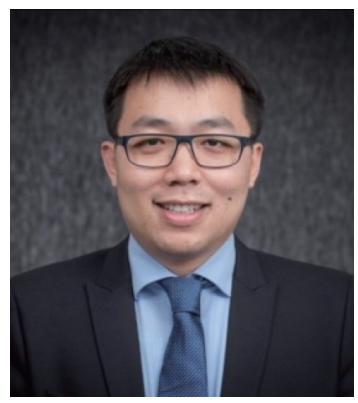

Dr. Huan Meng is a professor at the National Center for Nanoscience and Technology (NCNST) in China. He received his B.S. degree at Peking University and Ph.D. degree at Chinese Academy of Sciences, respectively. Before joining NCNST, he was an associate professor of David Geffen School of Medicine at University of California, Los Angeles (UCLA), and a faculty member in the Jonsson Comprehensive Cancer Center and California NanoSystems Institute. He has extensive experience in pharmaceutical science, nanomedicine, nanobiology, and drug delivery, focusing on the safe design of multifunctional nanocarriers, including the development of smart design features such as coating with synthetic or natural lipid bilayers, high drug loading capacity, self-assembly, circulatory stability, placement of on-demand delivery features, and imaging. 


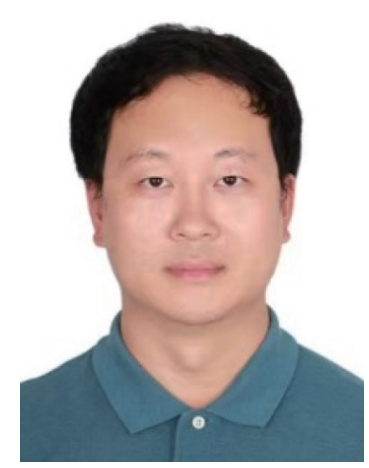

Dr. Lei Meng received his B.S. degree from Shanghai Jiao Tong University in 2011, M.S. degree from Northwestern University in 2012 and Ph.D. degree from University of California, Los Angeles (UCLA) in 2017 (advisor: Prof. Yang Yang), respectively. After graduation, he continued his postdoctoral research in UCLA. In 2018, he joined the Institute of Chemistry, Chinese Academy of Sciences as a professor in the Key Laboratory of Organic Solids. His current research interest mainly focuses on organic semiconductor materials, organic solar cells and perovskite solar cells. Since joined ICCAS, he has made progress in tandem organic solar cells and the enhancement of stability of perovskite solar cells.

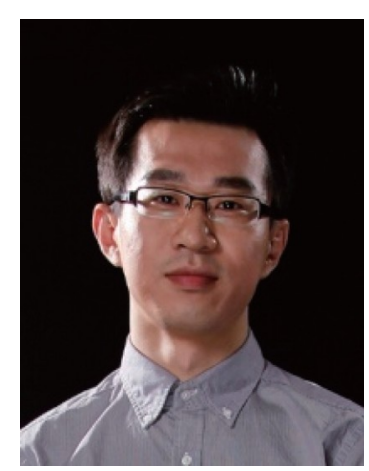

Dr. Zhiqiang Niu received his B.S. degree and Ph.D. degree from Peking University in 2007 and Tsinghua University in 2012, respectively. He carried out postdoctoral studies at Tsinghua University and University of California Berkeley in 2012-2017. In the meantime, he also worked in California Research Alliance (CARA) by BASF on transparent conductors. After having some experience in industry in 20172019, he joined the faculty in the department of chemical engineering at Tsinghua University in 2019. His current research interest focuses on the development of advanced catalytic materials for electrochemical energy conversions, and the study of the underlying mechanisms.

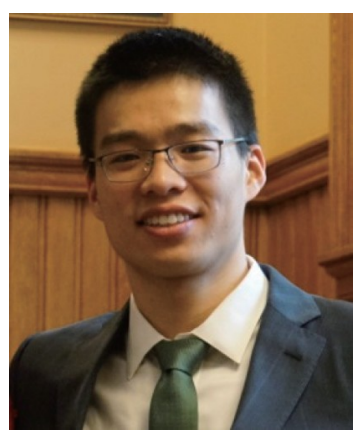

Dr. Quanquan Pang is currently an assistant professor in the School of Materials Science and Engineering at Peking University. He received his Ph.D. degree in Chemistry under the supervision of Prof. Linda F. Nazar from University of Waterloo in 2017, working on the many aspects of lithiumsulfur batteries. He then completed his postdoctoral research at Massachusetts Institute of Technology before joining Peking University in 2020. His current research interests include the materials chemistry and electrochemistry in a variety of energy storage systems, including metal-sulfur batteries, multi-valent batteries, and solid-state batteries.

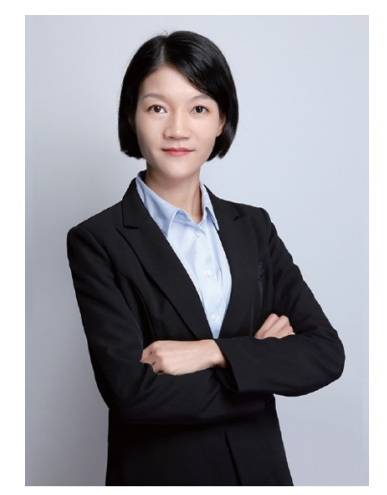

Dr. Liping Qiu received her Ph.D. degrees in analytical chemistry from Hunan University, and began her academic career at the same university in 2014. Within her Ph.D education, she spent two years, as a visiting student, at University of Florida, USA. From 2015 to 2016, she worked as a postdoctor at the Tumor Immunology department of Radboud University Medical Center, the Netherlands. In 2017, she was selected as an Alexander von Humboldt Fellow and worked at Bundesanstalt für Materialforschung und -prüfung, Germany. She has long been engaged in interdisciplinary research of analytical chemistry and biomedicine. Her research interest includes biosensing and biomanipulation, live-cell imaging, and tumor immunology. 


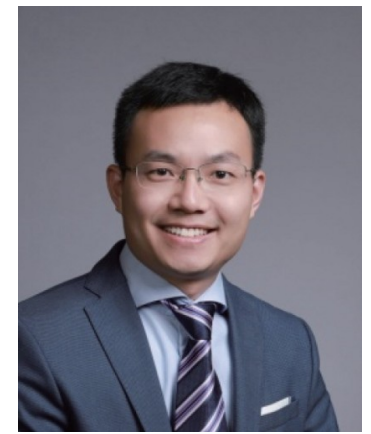

Dr. Hairen Tan has been a full professor in the College of Engineering and Applied Sciences at Nanjing University since 2018. He obtained his bachelor degree of engineering in Materials Science from Central South University in 2008, master degree of science from the Institute of Semiconductors of CAS in 2011, Ph.D. degree from Delft University of Technology in 2015, respectively. After Ph.D. graduation, he was awarded the Rubicon Fellowship from NWO and joined Ted Sargent's group at University of Toronto as a postdoctoral fellow during 2015-2018. His current research focuses on developing high-efficiency and costeffective photovoltaic devices, including perovskite solar cells, silicon solar cells, and perovskite-based tandem solar cells. He serves as the external editor of Commun. Mater., and the editorial board member of J. Semicond. and Sci. China Mater..

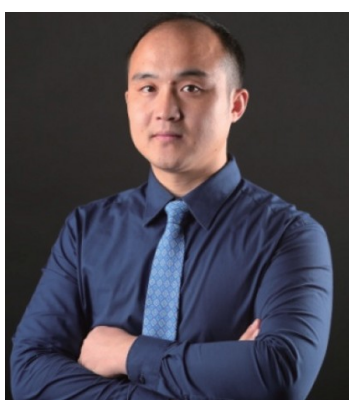

Dr. Dong Wang received his Ph.D. degree from Bordeaux University, and he conducted his postdoctoral study at the University of Toronto and The Hong Kong University of Science and Technology (HKUST). He is currently an associate professor and executive director of Center for AIE Research in Shenzhen University. His research focuses on the design of novel luminogens with aggregation-induced emission (AIE) features for theranostic applications.

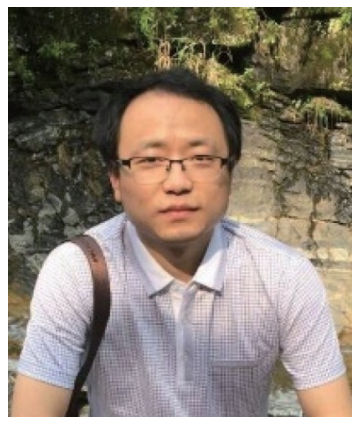

Dr. Chong Xiao obtained his Ph.D. degree from the University of Science and Technology of China (USTC) in 2013. $\mathrm{He}$ is now a professor of Hefei National Laboratory for Physical Sciences at Microscale, USTC. He has been working on advanced energy conversion materials over ten years, from materials synthesis and characterization to understanding the underlying physics and chemistry mechanism. His recent research interest focuses on the design and synthesis of low-dimensional inorganic solids with efforts to modulate their electron and phonon structures for energy storage and conversion applications.

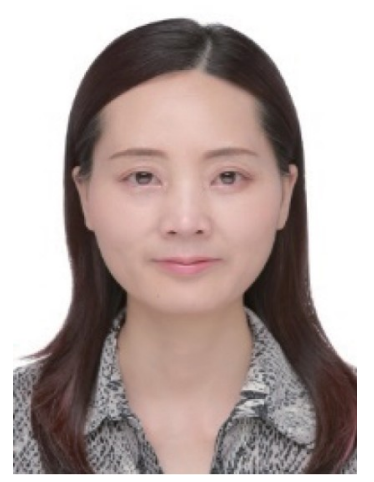

Dr. Haiyan Xie obtained her Ph.D. degree from Wuhan University in 2004. She then joined the Beijing Institute of Technology (BIT) and started her independent research. In 2013, she worked as a visiting scholar in the School of Medicine in Stanford University. Her current research interest is bioimaging analysis and nano-biomedicine. She is the recipient of the National Science Fund for Distinguished Young Scholars of China (2020), the National Funds for Distinguished Young Scientists (2014), the Program of New Century Excellent Talents of the Ministry of Education, and the Program of Beijing Excellent Talents (2008). 


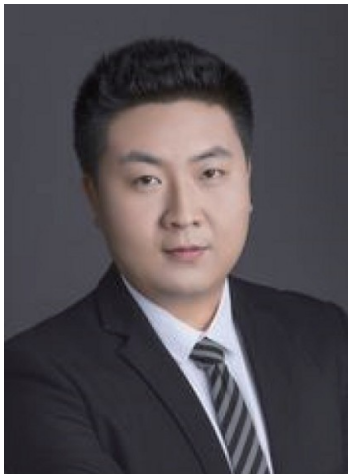

Dr. Jin Xie received his Ph.D. degree degree in 2013 from Nanjing University under the supervision of Prof. Chengjian Zhu. From 2014-2017, he was a postdoctoral research associate in the group of Prof. A. S. K. Hashmi at Heidelberg University. In 2017, he came back to Nanjing University to start his independent career and was appointed as the principal investigator. His current research interests lie in bimetallic chemistry and synergistic radical chemistry.

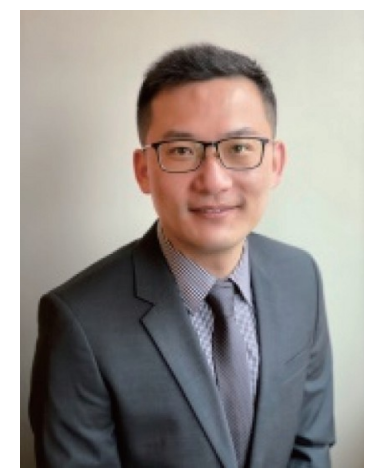

Dr. Yang $\mathrm{Xu}$ is an assistant professor in electrochemical energy storage in the Department of Chemistry at University College London (UCL), UK. He received his bachelor's (2006) and Ph.D. degrees (2011) at the University of Science and Technology of China. He carried out postdoctoral research on solar water splitting at Boston College (USA) and $\mathrm{Na}$-ion batteries at the University of Alberta (Canada). He then took on a Senior Scientist role at Technical University of Ilmenau (Germany), where he continued research on $\mathrm{Na}$ ion batteries and pioneered to K-ion batteries. In April 2019, he was appointed a faculty position at UCL. The research in his group focuses on beyond-Li battery chemistries ( $\mathrm{Na}, \mathrm{K}$, $\mathrm{Mg}$ and $\mathrm{Ca}$ ), spanning from the design of electrode materials with structural defects and low crystallinity to the associated ion transport and storage mechanism, and further to surface electrochemical reactivity at solid-liquid interfaces in nonaqueous and aqueous electrolytes.

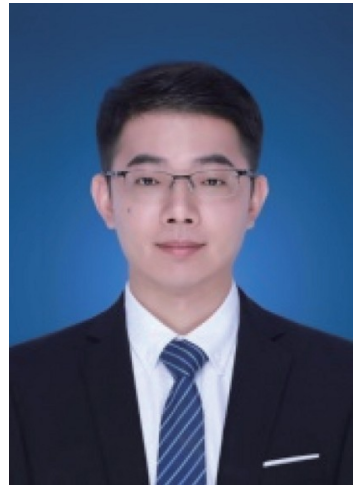

Dr. Long Ye is a full professor at the School of Materials Science \& Engineering of Tianjin University. He received his Ph.D. degree from the Institute of Chemistry, Chinese Academy of Sciences in 2015. From 2015 to 2019, he was a postdoctoral researcher and later promoted to research assistant professor at the Department of Physics, North Carolina State University, United States. Then, he started his independent career at Tianjin University in October, 2019. His current research interest includes morphological, thermal, and mechanical characterizations of semiconducting polymers and their blends, and polymer physics of conjugated polymers that can help tackle the challenges in organic solar cells, transistors, and photodetectors.

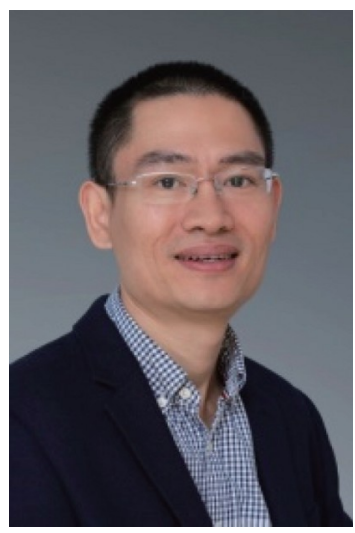

Dr. Long-Wu Ye received his B.Sc. degree in chemistry from Zhejiang University (2003). He then obtained his Ph.D. degree in 2008 from Shanghai Institute of Organic Chemistry, CAS, under the guidance of Prof. Yong Tang. After postdoctoral studies with Prof. Cheng Liu and Subhash Sinha at the Scripps Research Institute and with Prof. Liming Zhang at the University of California at Santa Barbara, he joined Xiamen University in 2011 as an associate professor. He was promoted to a full Professor in 2012. He was awarded National Natural Science Funds for Excellent Young Scholars (2016), Changjiang Young Scholars (2017), National Natural Science Funds for Distinguished Young Scholars (2021), etc. His research interest mainly focuses on catalytic asymmetric reactions based on alkynes and their use in $N$-heterocycle synthesis. 


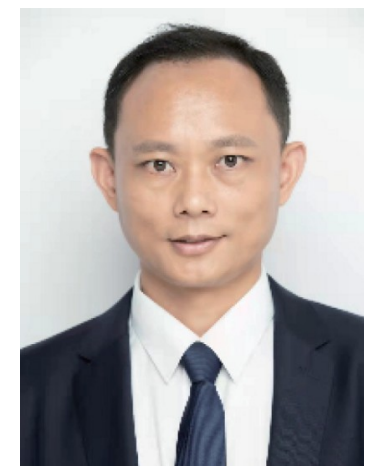

Dr. Mengchun Ye received his B.Sc. degree from Lanzhou University in 2001 and Ph.D. degree from the Shanghai Institute of Organic Chemistry, CAS under the supervision of Professor Yong Tang in 2006. After three-year postdoc experience on drug discovery in the Professor Hengming Ke's laboratory at University of North Carolina at Chapel Hill, he moved to the Scripps Research Institute in 2009, where he worked on $\mathrm{C}-\mathrm{H}$ activation with Professor Jin-Quan Yu. In 2014, he joined the faculty of the College of Chemistry at Nankai University. His current research focuses on inert chemical bond activation and synergistic catalysis.

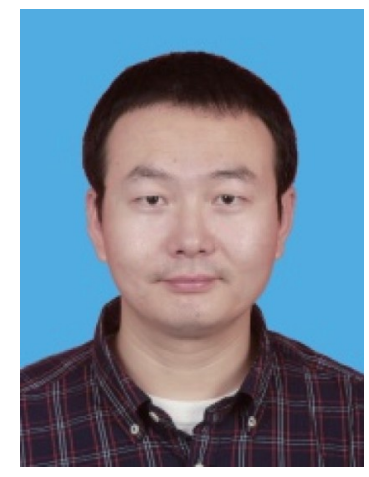

Dr. Lei Ying received his Ph.D. degree from South China University of Technology (SCUT) in 2009 under the supervision of Prof. Wei Yang. After post-doctoral research in University of California Santa Barbara with Prof. Guillermo C. Bazan, he started his academic carrier at Institute of Polymer Optoelectronic Materials and Devices at SCUT in 2013. He was promoted to full professor in 2017. His current interest is developing new semiconducting polymers and extending their applications in optoelectronic devices. He is also a co-founder of Dongguan Volt-Ampere Optoelectronics Co., LTD.

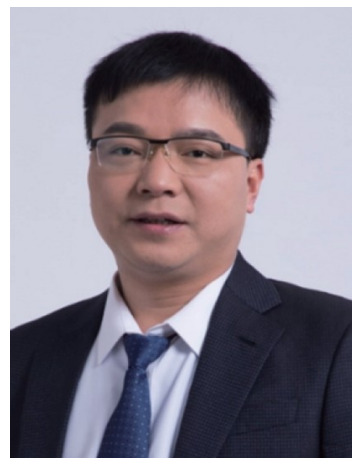

Dr. Zebing Zeng obtained his bachelor degree from Hunan University of Technology in 2004, master degree from Nankai University in 2007, and Ph.D. degree in 2012 at National University of Singapore (NUS). After two-year postdoctoral research in NUS, he commenced his research activities as an associate professor of Hunan University at College of Chemistry and Chemical Engineering in 2014, and was promoted to full professor in 2019. During 2016, he was a half-year visiting scientist at the Hong Kong University of Science and Technology. His research interest includes novel $\pi$-conjugated and radical-based organic materials with intriguing optoelectronic and magnetic properties as well as at aggregation level.

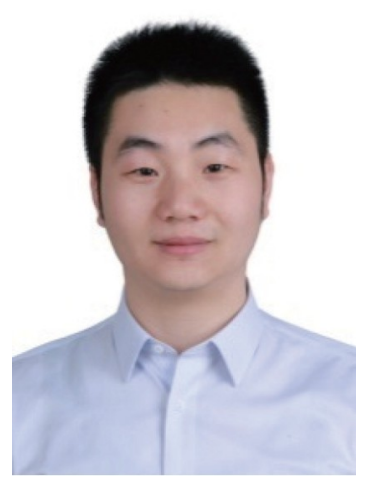

Dr. Xiaodong Zhang received his B.S. degree from Anhui University in 2008 and Ph.D. from the USTC in 2013. He has then worked as a postdoctoral fellow in Collaborative Innovation Center of Chemistry for Energy Materials (iChEM). He started his academic career as an assistant professor at USTC in 2015, where he is a full professor now. His research interest focuses on low-dimensional photoresponsive materials and regulation of their intrinsic physical properties for functional applications, which promotes the development of excitonic material chemistry. 